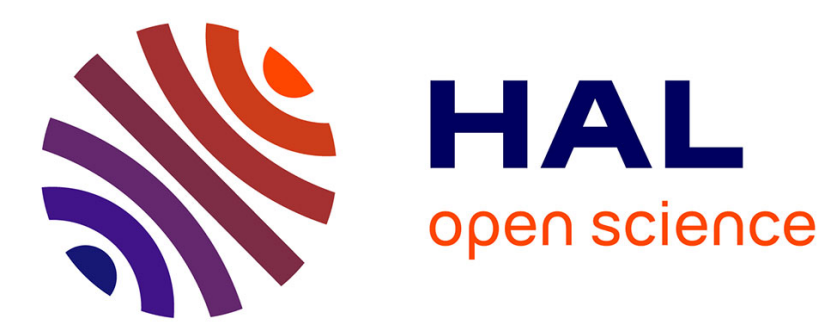

\title{
Dispositif de mesure de la magnétostriction en longueur
}

Roger Vautier, Étienne de Lacheisserie

\section{To cite this version:}

Roger Vautier, Étienne de Lacheisserie. Dispositif de mesure de la magnétostriction en longueur. Revue de Physique Appliquée, 1966, 1 (1), pp.37-42. 10.1051/rphysap:019660010103700 . jpa-00242682

\section{HAL Id: jpa-00242682 https://hal.science/jpa-00242682}

Submitted on 1 Jan 1966

HAL is a multi-disciplinary open access archive for the deposit and dissemination of scientific research documents, whether they are published or not. The documents may come from teaching and research institutions in France or abroad, or from public or private research centers.
L'archive ouverte pluridisciplinaire HAL, est destinée au dépôt et à la diffusion de documents scientifiques de niveau recherche, publiés ou non, émanant des établissements d'enseignement et de recherche français ou étrangers, des laboratoires publics ou privés. 


\title{
DISPOSITIF DE MESURE DE LA MAGNÉTOSTRICTION EN LONGUEUR
}

\author{
Par Roger VAUtier et Étienne DE LACHEISSERIE, \\ Laboratoire de Magnétisme et de Physique du Solide, C. N. R. S., Bellevue (Hauts-de-Seine).
}

Résumé. - On décrit un dilatomètre à pont de capacités, dont la caractéristique $\Delta C=f(\Delta l)$ est parfaitement linéaire pour tout déplacement de l'électrode mobile inférieur à 100 microns. Ce dispositif permet de mesurer la magnétostriction longitudinale d'échantillons de $1 \mathrm{~cm}$ de long dans la gamme $10^{-8}$ à $5 \times 10^{-3}$, dans un champ de $9 \mathrm{kOe}$, et à toutes températures jusqu'à $900{ }^{\circ} \mathrm{K}$.

Abstract. - A capacitor bridge dilatometer is described ; its characteristic $\Delta C=f(\Delta l)$ is perfectly linear for any displacement of the moving electrode less than $100 \mu \mathrm{m}$. This device allows us to measure the longitudinal magnetostriction of one centimeter samples. in the range from $10^{-8}$ to $5 \times 10^{-3}$, in a field strength up to $9 \mathrm{kOe}$ and at any temperature below $900 \mathrm{oK}$.

I. But et principe de l'appareil. - L'appareillage décrit est destiné à la mesure de la magnétostriction en longueur sur des échantillons de dimensions de l'ordre du centimètre. L'effet en question consiste en une variation des dimensions d'un échantillon ferromagnétique ou ferrimagnétique lorsqu'on le soumet à un champ magnétique. Cette variation est toujours très petite, au maximum de $10^{-3}$ en valeur relative et de l'ordre de $10^{-5}$ à $10^{-6}$ dans la plupart des cas. Sa mesure nécessite donc un matériel très sensible.

Beaucoup d'études actuelles de magnétostriction sont faites à l'aide de jauges résistantes qui présentent des avantages certains. Mais nous avons pensé que des renseignements précieux pourraient être obtenus en employant concurremment une méthode différente. En effet, les résultats de ce genre de mesure sont souvent délicats à interpréter, et la comparaison des courbes obtenues par deux méthodes différentes doit permettre de mieux les comprendre, et en particulier de séparer plus facilement les phénomènes imputables à l'échantillon lui-même et ceux éventuellement dus aux appareillages de mesure.

Le dispositif que nous avons réalisé et dont la figure 1 donne une vue d'ensemble comporte un transducteur, composé de quatre capacités variables montées en pont ( $f g .2)$, dont l'équilibre est modifié par le déplacement à mesurer, et une chaîne de mesure (fig. 3) chargée de fournir la tension d'attaque du pont et d'amplifier le signal de sortie. Contrairement à la plupart des dispositifs capacitifs utilisés dans le passé (voir par exemple [1] et [2]), cet appareil assure une linéarité parfaite de la fonction $\Delta C I C=f(\Delta l / l)$ pour des variations relatives de longueur de $\pm 10^{-8}$ à $\pm 10^{-2}$.

II. Description des éléments. - II-1. Le transDucteur. - Les électrodes des quatre capacités variables sont constituées par un dépôt d'or évaporé

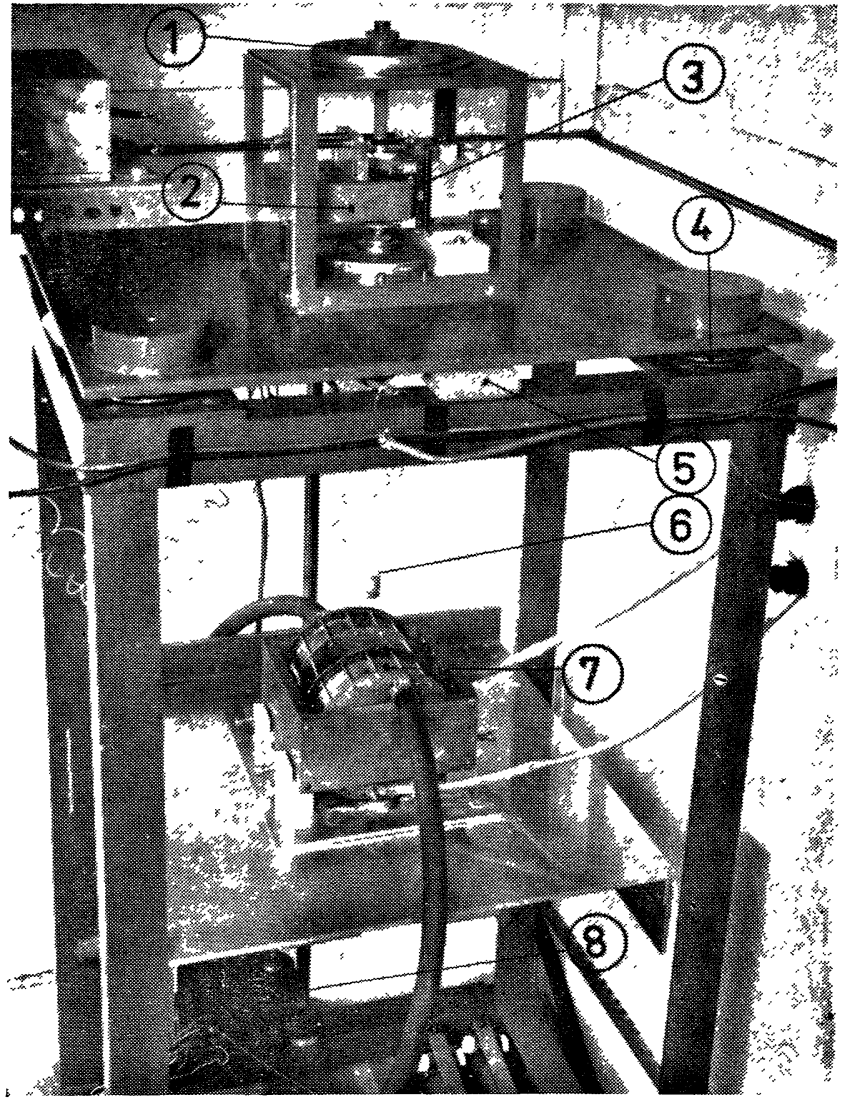

Frg. 1. - Vue d'ensemble de l'appareil. 1. Membrane de suspension de la tige en quartz. - 2. Boîtier du préamplificateur. - 3. Les plaques du condensateur. - 4. Suspension élastique du chàssis de mesure. - 5. Amortisseur. - 6. Échantillon. 7. Bobine de mesure de la magnétostriction transversale. - 8. Moteur permettant d'amener le centre magnétique de la bobine en coïncidence avec le centre de gravité? de l'échantillon. 


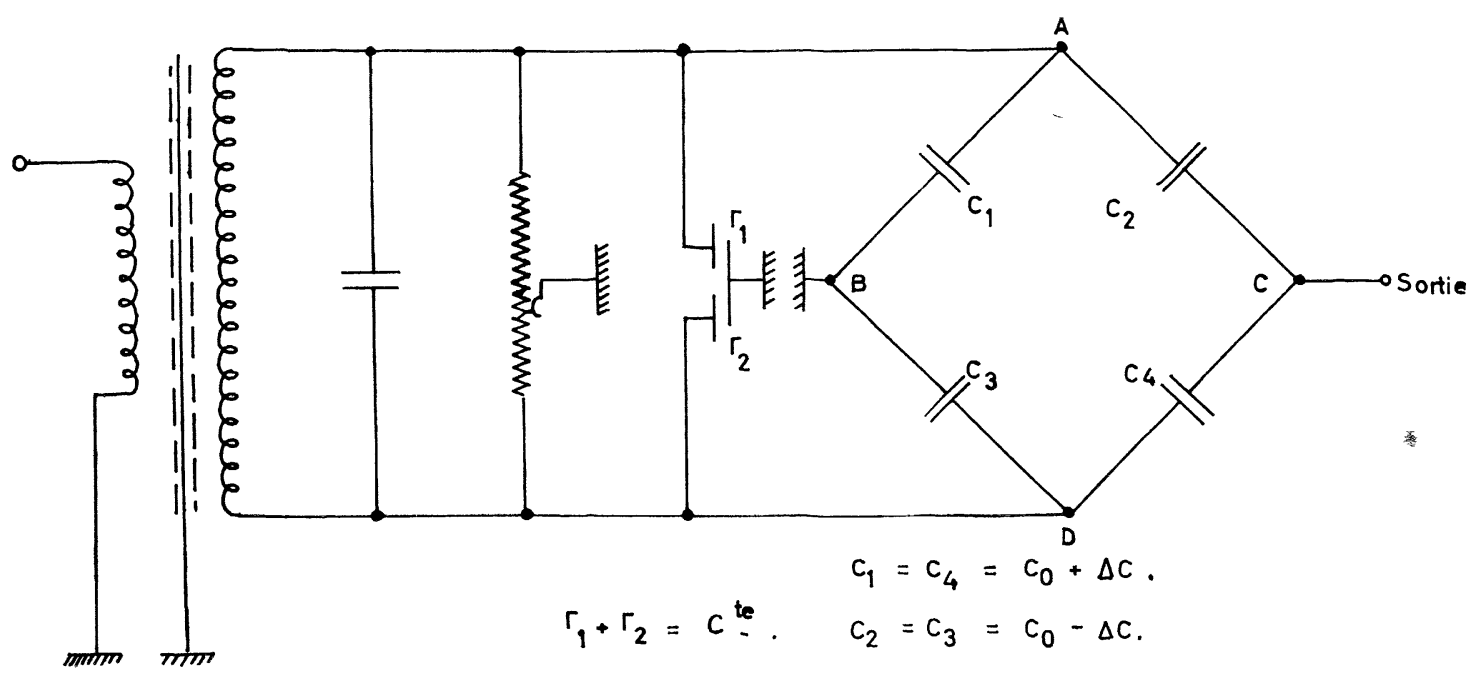

FIg. 2. - La tête de pont et le transducteur. $\Gamma_{1}+\Gamma_{2}=$ Cte. $C_{1}=C_{4}=C_{0}+\Delta C \quad C_{2}=C_{3}=C_{0}-\Delta C$.

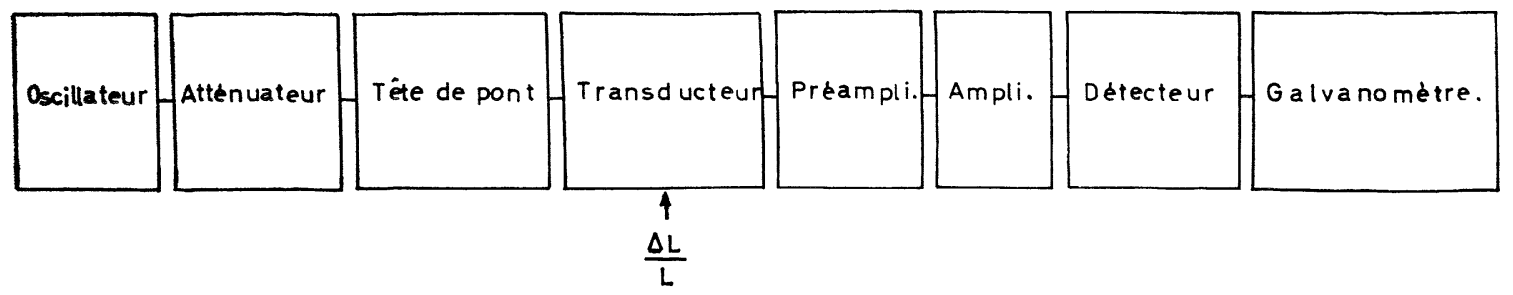

FIg. 3. - Schéma bloc de la chaîne_de mesure.

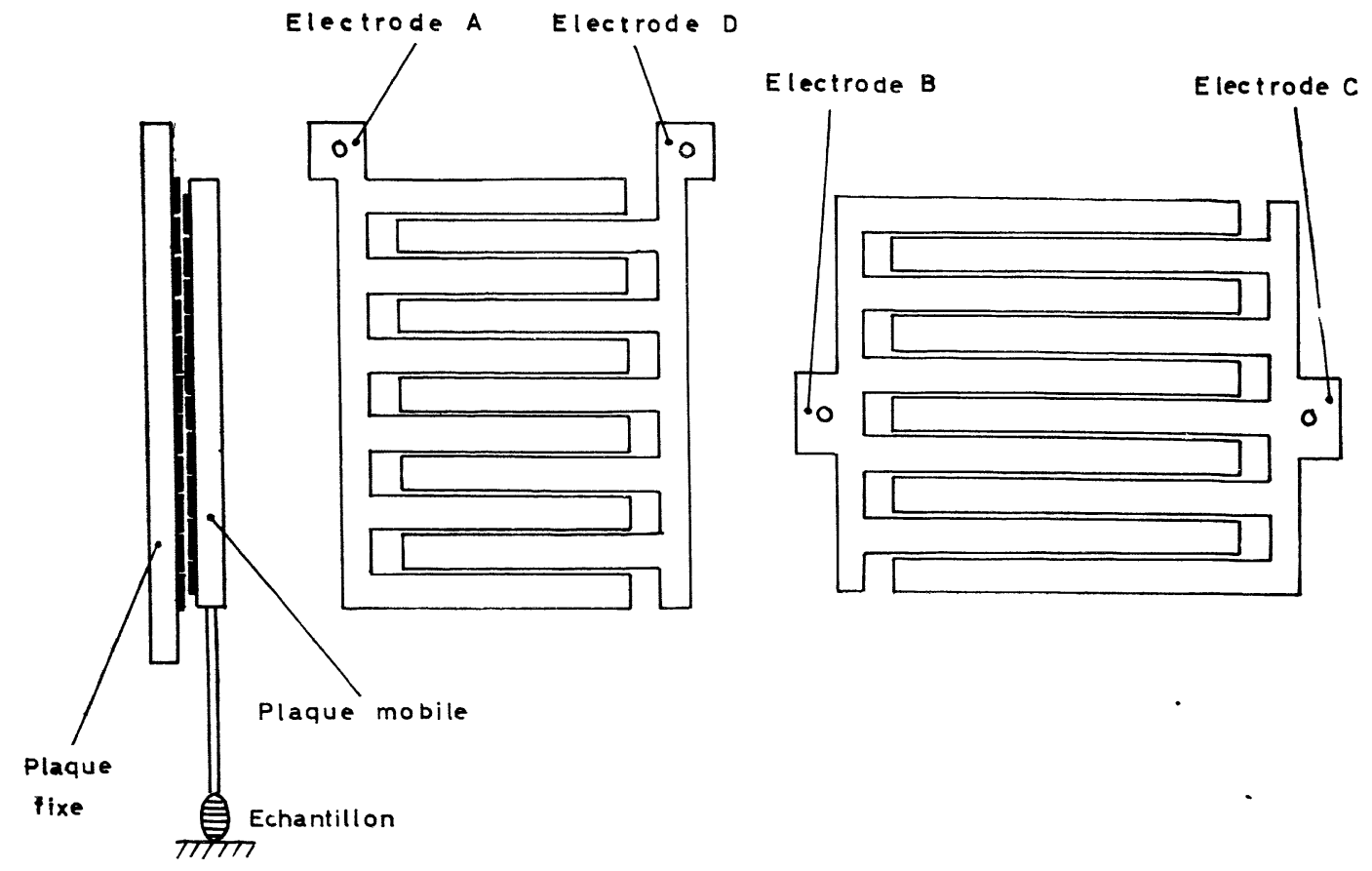

FIG. 4. - La disposition des électrodes. 
sous vide sur deux plaques en silice transparente; chaque plaque mesurant $8 \mathrm{~cm} \times 6 \mathrm{~cm}$ porte deux électrodes, elles-mêmes constituées chacune de 32 électrodes élémentaires fonctionnant en parallèle. L'une des plaques est fixe ; l'autre est solidaire d'une tige en silice qui repose sur l'extrémité supérieure de l'échantillon. Cette tige est portée par deux membranes élastiques qui assurent un déplacement bien vertical. La distance entre les deux plaques est $0,2 \mathrm{~mm}$ environ ; la valeur des capacités à l'équilibre est alors $C_{0}=30 \mathrm{pF}$. La figure 4 représente très schématiquement la disposition adoptée. Les matériaux employés ont été choisis en vue de compenser les effets de la dilatation thermique. De plus, un blindage thermique efficace constitué par des panneaux de polystyrène expansé maintient l'ensemble à température constante, et le protège aussi des bruits extérieurs qui se traduiraient par des vibrations parasites des plaques du condensateur et réduiraient donc la sensibilité de l'appareil. Enfin la suspension du plan de référence comporte des liaisons élastiques et des amortisseurs, destinés à atténuer les vibrations transmises par le sol ( $\mathrm{fg} .1$ ).

L'extrémité inférieure de l'échantillon est solidaire d'un tube en silice concentrique à la tige mobile et fixé par une pince au plan de référence. Le mode de fixation de l'échantillon joue un grand rôle dans la précision de la mesure : la liaison doit être rigide à toutes les températures de travail, et néanmoins ne pas affecter les propriétés physiques du corps étudié. Deux types de fixation se sont révélés commodes :

- la fusion d'une goutte de gomme laque au pied de l'échantillon, posé sur une vis en céloron ( $f g .5 a$ ). Un petit montage annexe maintient l'échantillon bien vertical au cours de la fusion qui s'opère en étuve à $120^{\circ} \mathrm{C}$;

- l'introduction de l'échantillon taillé sous forme de cylindre de diamètre $1,9 \mathrm{~mm}$ dans un orifice cylindrique de diamètre $1,85 \mathrm{~mm}$ et hauteur $1,2 \mathrm{~mm}$ percé dans une vis en laiton ; celle-ci est fendue pour être rendue élastique ( $f g .5 b)$.

Dans les deux cas, il ne reste plus qu'à fixer au tube en quartz la vis solidaire de l'échantillon, par l'intermédiaire d'une bague filetée. La fixation par gomme laque fournit les meilleurs résultats pour les températures inférieures à $+50^{\circ} \mathrm{C}$, en particulier pour les métaux tels que le nickel, qui sont très sensibles aux tensions. C'est par contre la seconde solution qui s'impose lorsqu'il s'agit d'effectuer des mesures à haute température, jusqu'à $500^{\circ} \mathrm{C}$; dans le cas des ferrites, il est apparu que ceux-ci sont peu sensibles à la contrainte mécanique que la pince exerce sur le bout de l'échantillon.

II-2. La CHAINe de mesure. - Elle comporte un oscillateur stable à $455 \mathrm{kHz}$ délivrant une tension de 2 volts efficaces sur $50 \Omega$, suivi d'un atténuateur de 0 à $80 \mathrm{~dB}$, par plots de $10 \mathrm{~dB}$. Des éléments de

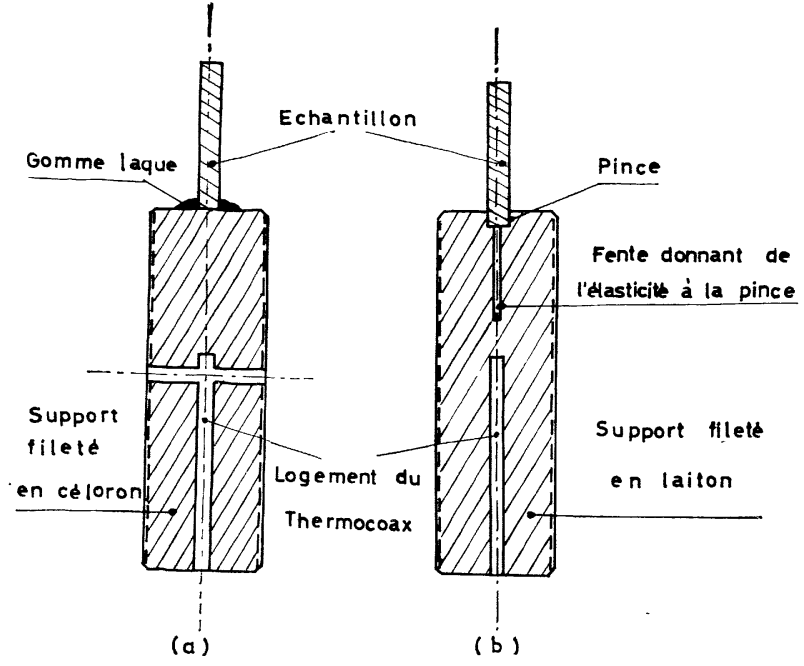

Fig. 5. - Porte-échantillons : $(a)$ pour basses températures ; $(b)$ pour hautes températures.

réglage capacitifs et résistifs, qui constituent la " tête de pont ", permettent de corriger les déséquilibres du pont, correspondant à des déplacements inférieurs à $50 \mu$. Les capacités $\Gamma_{1}$ et $\Gamma_{2}$ sont réglables, mais leur somme reste constante (fig. 2 ). Le schéma électrique du transducteur se réduit à quatre capacités variables : $C_{1}$ et $C_{4}$ augmentant lorsque $C_{2}$ et $C_{3}$ diminuent. Un préamplificateur à transistors soigneusement blindé recueille le signal dès sa sortie du pont sous haute impédance, et le délivre sous $50 \Omega$ à un amplificateur de tension à grand gain. Après détection, le signal est lu sur un galvanomètre directement gradué en angströms. L'atténuateur d'entrée permet de toujours faire travailler la chaîne amplificatrice dans la même gamme de tensions : $0-20 \mu \mathrm{V}$ eff. à l'entrée du préamplificateur; sur la gamme la plus sensible (0 dB), la déviation totale du galvanomètre correspond à 50 Angströms.

¿II-3. LA PRODUCTION DU CHAMP MAgNÉTIQUE. Pour mesurer la magnétostriction transversale, nous disposons d'un petit électroaimant à fer qui produit environ $540000 \mathrm{At} / \mathrm{m}(\simeq 7 \mathrm{kOe}$ ), homogène à $1 \%$ sur $1 \mathrm{~cm}$, ce qui suffit pour les mesures de magnétostriction à saturation.

La géométrie de l'appareil interdisant l'emploi d'une bobine à fer pour créer un champ parallèle à la direction de mesure, un plus grand nombre d'Ampère-tours est alors nécessaire. Nous avons réalisé une bobine qui fournit un champ de $700000 \mathrm{At} / \mathrm{m}(\simeq 9 \mathrm{kOe})$, quand elle est alimentée sous 220 volts. Elle dissipe alors $18 \mathrm{~kW}$. Le refroidissement du bobinage est assuré par une circulation d'eau : chaque couche est séparée de la suivante par des barrettes en téflon et les joues sont finement découpées pour diminuer au maximum la 
perte en charge (fig. 6), suivant une technique utilisée depuis longtemps dans notre Laboratoire [3]. Le champ obtenu est constant à mieux que $\pm 0,5 \%$ sur $1 \mathrm{~cm}$.

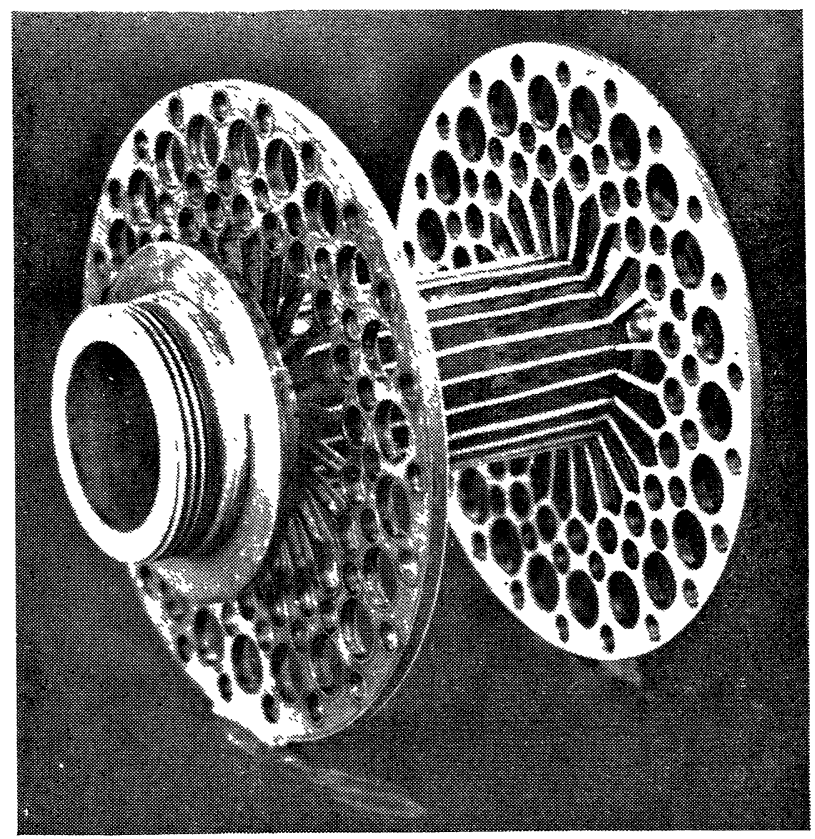

FIg. 6. - Carcasse de la bobine d'aimantation.

II-4. Les mesures en température. - Pour effectuer les mesures à basse température, on plonge le tube en quartz contenant l'échantillon dans un vase Dewar rempli d'azote, dont l'évaporation est assez lente pour permettre de mesurer $\lambda_{\mathrm{s}}$ à $\pm 3 \%$ entre $-196^{\circ} \mathrm{C}$ et $+20^{\circ} \mathrm{C}$. Pour obtenir les hautes température, un four anti-inductif de très faible encombrement remplace le vase Dewar. L'énergie dissipée compensant les pertes par rayonnement, la dérive thermique peut être suffisamment réduite pour permettre de lire $\lambda_{\mathrm{s}}$ à $\pm 3 \%$ de $+20^{\circ} \mathrm{C}$ à $+500^{\circ} \mathrm{C}$.

La température de l'échantillon est lue au moyen d'un Thermocoax logé dans le support de l'échantillon.

III. La technique de mesure.- III-1. L'ÉtALONNAGE. - En l'absence d'échantillon, la plaque mobile du condensateur est seulement portée par deux membranes élastiques circulaires. L'application d'une faible surcharge sur l'équipage mobile crée donc un déplacement proportionnel à cette charge, et le facteur de proportionnalité du déplacement à la force a été déterminé expérimentalement à $\pm 2 \%$ : pour étalonner l'appareil il suffit de placer sur l'équipage mobile une masse de $7,35 \mathrm{~g}$ ce qui abaisse l'ensemble de 5 microns. Un potentiomètre permet d'ajuster le gain de l'amplificateur, afin d'amener le spot du galvanomètre devant la division correspondante.

III-2. Les sources D'ERREUR. - L'application du champ magnétique introduit généralement des forces et des couples parasites qui peuvent masquer totalement les déformations dues à la magnétostriction. Ces sources d'erreur peuvent expliquer en partie la dispersion des résultats obtenus dans le passé avec des dilatomètres :

Couple exercé sur un échantillon mal orienté : Lorsqu'un échantillon allongé n'est pas correctement orienté par rapport au champ appliqué, il est soumis à un couple proportionnel à ce champ et à son aimantation; il en résulte une rotation de l'échantillon indiscernable du phénomène à mesurer et qui perturbe la mesure de la magnétostriction. Les dispositifs de fixation de l'échantillon décrits au paragraphe II-1. nous ont permis d'éliminer cette source d'erreur en imposant à l'échantillon une orientation correcte.

Si de plus l'échantillon est trop mince et n'est pas rigoureusement vertical, il peut fléchir sous la pression que lui communique la tige en quartz. Le calcul et l'expérience ont montré que cet effet ne peut pas jouer de façon appréciable sur des échantillons de $15 \mathrm{~mm}$ de long dont le diamètre est supérieur à $1,5 \mathrm{~mm}$.

Forces liées à l'inhomogénéité du champ extérieur : Le gradient du champ magnétique créé par nos bobines ne s'annulant qu'en leur centre magnétique, il en résulte l'apparition en tout point de l'échantillon de forces proportionnelles au champ. Nous avons calculé ces forces et évalué les déformations qui en résultent : elles sont minimales lorsque le centre de gravité de l'échantillon se trouve exactement situé au centre magnétique de la bobine. Cependant même dans ce cas se produit une légère contraction parasite, fonction linéaire du champ magnétique ; cet effet déjà calculé par Nagaoka [4] n'introduit pas d'erreur décelable lorsque la longueur de l'échantillon n'excède pas un centimètre, pour les bobines que nous utilisons. Par contre, dès que l'échantillon n'est plus exactement centré dans la bobine, il est soumis à une force puissante qui engendre une déformation supplémentaire pouvant fausser totalement la mesure de la magnétostriction forcée. C'est pourquoi nous avons dû équiper l'appareil d'un dispositif annexe permettant d'ajuster à $\pm 0,2 \mathrm{~mm}$ près la position relative de l'échantillon ct de la bobine.

Influence du champ magnétique sur le préamplificateur: Initialement, le tube et la tige en quartz utilisés étaient assez courts, et le préamplificateur (2, fig. 1) n'était situé qu'à $15 \mathrm{~cm}$ de la partie supérieure de la bobine. Sous l'influence du champ magnétique, nous observions une modification des caractéristiques des transformateurs MF à noyaux en ferrite, ce qui entraînait une variation du gain du 
préamplificateur. Il a suffi de rallonger le tube et la tige de $15 \mathrm{~cm}$ pour éliminer totalement cet effet parasite.

Couple créé par l'anisotropie du matériau: En prenant toutes les précautions nécessaires, il est théoriquement possible d'éliminer les phénomènes parasites décrits précédemment ; il n'en est pas de même pour les forces liées à l'anisotropie du matériau, qui exercent sur l'échantillon un couple dont il est impossible de s'affranchir : l'étude de la magnétostriction forcée d'un monocristal de nickel taillé dans le plan (110) nous a fourni, pour un champ parallèle aux directions [110], [111] et [001], des résultats reproductibles et en accord avec ceux que d'autres chercheurs ont obtenu par la méthode des jauges [5] ; par contre pour toutes les autres directions, pour lesquelles le couple d'anisotropie n'est pas nul, nous avons obtenu des résultats peu reproductibles et supérieurs à ceux que le calcul laissait prévoir. Seule, semble-t-il, la technique des jauges permet de limiter les effets fâcheux de l'anisotropie, car il est possible d'utiliser une jauge de dimensions faibles vis-à-vis du diamètre de l'échantillon, et done moins sensible aux déformations qui affectent surtout le périmètre du cristal, au voisinage des points de fixation.

III-3. Les Performances obtenues. - Les vibrations résiduelles transmises par le sol et l'atmosphère limitent la sensibilité de l'appareil à $\pm 10-8$ d'allongement relatif sur un échantillon long de un centimètre. Dans les meilleures circonstances, la dérive thermique peut être réduite à 1 ou $2 \AA$ par minute. La précision relative et la reproductibilité des mesures d'allongements à déviation totale sont de 2 à $3 \%$ à toutes températures et $1 \%$ à la température ambiante. L'exactitude de l'étalonnage est du même ordre.

Il est possible en cours de mesure d'opérer un décalage de zéro en refaisant l'équilibre du pont, ce qui permet de repasser sur la gamme la plus sensible pour étudier avec précision la magnétostriction forcée.

Pour illustrer les possibilités de cet appareillage, nous donnons ( $f i g .7$ et 8 ) des résultats de mesures à la température ordinaire et à $407^{\circ} \mathrm{C}$. A la température ordinaire, on peut obtenir une précision très élevée car il est possible d'annuler pratiquement la dérive thermique (on peut la rendre $<$ à $1 \AA / \mathrm{mn}$ ).
La figure 7 représente la magnétostriction d'un échantillon de ferrite de composition $10 \mathrm{NiO}, 40 \mathrm{ZnO}$, $50 \mathrm{Fe}_{2} \mathrm{O}_{3}$ de $15 \mathrm{~mm}$ de long et $1,9 \mathrm{~mm}$ de diamètre. Ce ferrite est paramagnétique à la température

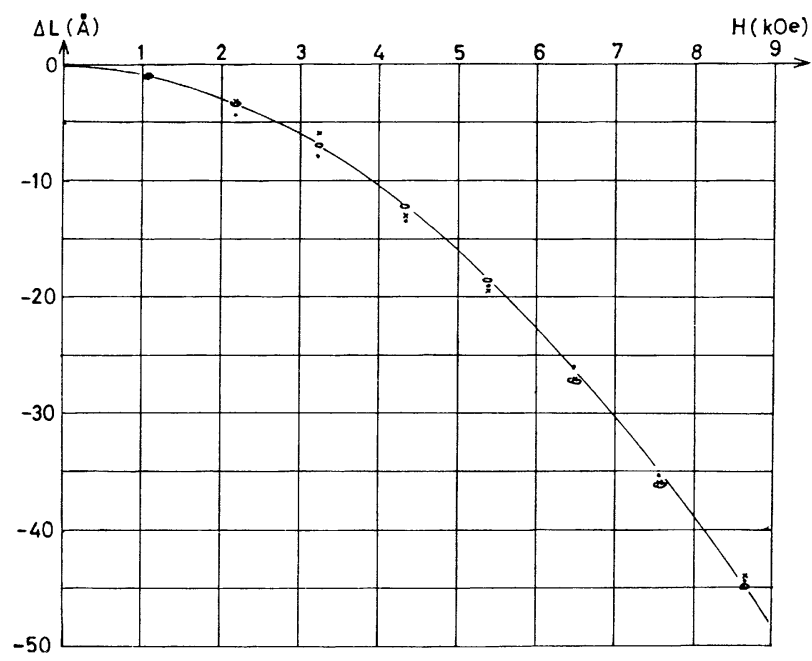

FIG. 7. - Une mesure de haute précision : la magnétostriction du ferrite $10 \mathrm{NiO}, 40 \mathrm{ZnO}, 50 \mathrm{Fe}_{2} \mathrm{O}_{3}$, paramagnétique à température ambiante.

ordinaire et ne présente alors qu'une très faible magnétostriction variant comme le carré du champ magnétique. Trois séries de mesures faites à deux mois d'intervalle ont vérifié cette loi à $\pm 3 \AA$ près.

A température élevée, la dérive est obligatoirement plus importante. La figure 8 est relative à un ferrite de nickel-zinc de composition $40 \mathrm{NiO}$, $10 \mathrm{ZnO}, 50 \mathrm{Fe}_{2} \mathrm{O}_{3}$. L'échantillon avait $17 \mathrm{~mm}$ de long et $1,9 \mathrm{~mm}$ de diamètre. Pour tenir compte de la dérive thermique on suppose que celle-ci reste une fonction linéaire du temps pendant la durée de la mesure (environ quarante secondes). La deuxième ligne du tableau I donne la lecture brute de $\Delta l$, la troisième ligne donne la valeur de $\Delta l$ corrigée de la dérive thermique.

La figure 8 représente en fonction du champ magnétique $H$ la valeur corrigée de $\Delta l / l$. Sur cette courbe, on opère la correction de magnétostriction forcée en extrapolant à champ interne nul, et on obtient ainsi la valeur $\lambda_{\mathrm{s}}$ de la magnétostriction à saturation soit :

$$
\lambda_{\mathrm{s}}=-(5,7 \pm 0,2) 10^{-6} \text { à } T=680^{\circ} \mathrm{K} .
$$

暴

$\begin{array}{lrrrrrrrrrrrr}H(\mathrm{Oe}) & 0 & 1080 & 2160 & 3240 & 4320 & 5400 & 5400 & 4320 & 3240 & 2160 & 1080 & 0 \\ \begin{array}{l}\Delta l(\AA) \\ \Delta l(\AA)\end{array} & 0 & -880 & -910 & -900 & -900 & -880 & -830 & -730 & -610 & -450 & -280 & +770 \\ \begin{array}{c}\Delta l(\AA) \\ \text { corrigé }\end{array} & 0 & -950 & -1050 & -1110 & -1180 & -1230 & -1250 & -1220 & -1170 & -1080 & -980 & 0 \\ -\frac{\Delta l}{l} 10^{6} & 0 & +5,6 & 6,2 & 6,5 & 6,9 & 7,2 & 7,4 & 7,2 & 6,9 & 6,35 & 5,8 & 0\end{array}$

\section{TABLEAU I}




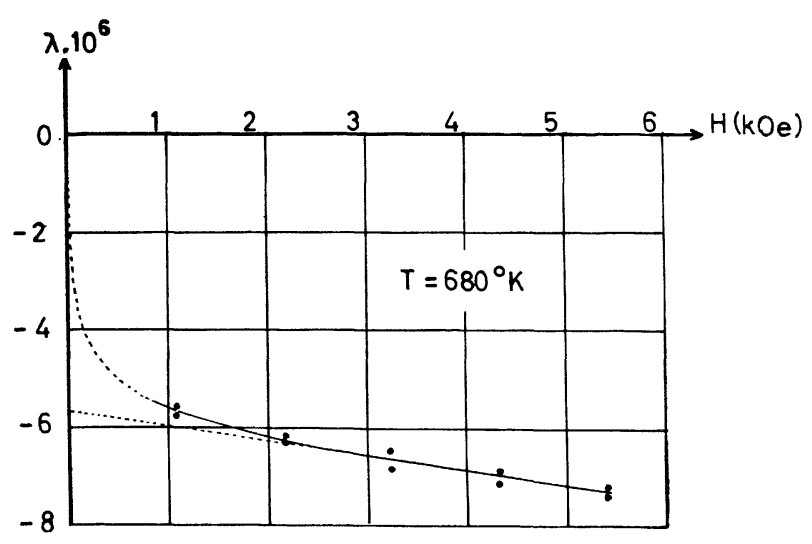

Fig. 8. - Un exemple de mesure à haute température.
Cet appareil d'un emploi facile permet donc la mesure d'allongements relatifs compris entre $\pm 10^{-8}$ et $\pm 5 \times 10^{-3}$ avec une précision de $2 \%$. Il se prête mieux que les jauges à des mesures de série ainsi qu'aux études en température de 0 à 900 o K ; les jauges sont par contre préférables pour la mesure de la magnétostriction forcée des échantillons magnétiquement anisotropes.

Remerciements. - Nous sommes heureux d'exprimer notre reconnaissance à $M$. Laporte à qui nous devons la réalisation particulièrement soignée de la partie mécanique de cet appareil.

Manuscrit reçu le 26 novembre 1965.

\section{RÉFÉRENCES}

[1] Dietsch (G.), Z. Techn. Physik, 1931, 8, S. 380.

[2] Corner (W. D.) et Hunt (G. H.), J. Sc. Instr., décembre 1954, 31, 12, 445-447.

[3] Vautier (R.), Thèse, Paris, 1954, p. 25.
[4] Nagaoka, Ann. Phys. Chemie, 1894, 53, 1493.

[5] Lourens (J. A. J.) et Alberts (L), Solid State Comm., 1964, 2, no 5, 141-143 